\title{
LOW PRESSURE CHEMICAL VAPOR DEPOSITION OF TIN OXIDE THIN FILMS FROM Al ORGANOMETALLIC COMPOUND. APPLICATION TO GAS DETECTION
}

\author{
C. PIJOLAT, L. BRUNO and R. LALAUZE \\ École Nationale Superieure des Mines de Saint-Etienne. \\ Département de Chimie-Physique des Processus Industriels, \\ 158 Cours Fauriel, F-42023 Saint-Etienne Cedex 2, France
}

\begin{abstract}
Tin dioxide $\mathrm{SnO}_{2}$ is one of the most common materials used in the fabrication of semiconductor gas sensors The principle of such sensors is based upon the variations of conductivity induced by adsorption of various gases.

A low pressure OMCVD apparatus has been developed in order to obtain tin dioxide thin films. Structural and electrical properties have been investigated by different techniques: S.E.M., X-ray diffraction and conductivity measurements in different atmospheres. A correlation is found between the deposition parameters and the electrical properties of CVD-SnO 2 thin films.
\end{abstract}

\section{Introduction}

In the fifties, it has been shown that adsorption of a gas on the surface of a semiconductor can bring about an important change in the electrical resistance of the material. The phenomenon has been applied to the detection of inflammable gases by Seiyama (1). Different oxides can be used $\left(\mathrm{TiO}_{2}, \mathrm{ZnO}, \mathrm{ZrO}_{2}, \ldots\right)$ but tin dioxide, because of its high sensitivity, is one of the most common materials used in the fabrication of semiconductor gas sensor. The first $\mathrm{SnO}_{2}$ gas sensor was marketed in 1968 (2). This sensor was developed for the detection of domestic gases. Actually, there are a lot of applications for gas sensors in many different fields (3): fire detectors, $\mathrm{CO}$ detectors for parking area, methane or propane sensors for domestic use, gas leak detection in industry, detection of neurotoxic gases, ...

The principle of semiconductor gas sensors is based upon variations of conductivity induced by the adsorption of various gases. The conductivity of the sensitive material is generally enhanced by reducing gases for n-type semiconductors (eg: $\mathrm{SnO}_{2}$ ) and by oxidizing gases for p-type semiconductors. All the phenomena which act on the electrical conductivity of such polycrystalline materials are dependent on the temperature: gas adsorption, catalytic activity, intrinsic resistance, grain boundaries barriers, electrical contacts, ... (4). In these conditions, conductance versus temperarature curves can exhibit maxima with some gases. There are principally two ways of working: the sensor can be used at the temperature of the maximum of conductance, or it can be used in temperature scanning. The difference in conductivity when the sensor is exposed to air or to a gas (even at low concentration of a few $\mathrm{ppm}$ ) allows one to detect the presence of that gas.

The principal characteristics of a good sensor are the sensitivity, the selectivity to different gases, and particulary the long-term stability. A lot of studies have shown that the sensitivity can be greatly improved by the addition of dopants.Concerning the selectivity, it is 
possible, in some cases, to use the properties of $G=f\left(T^{\circ}\right)$ curves having different maxima depending upon the nature of the gas, to detect a specific gas in a mixture.For example, thermal and chemical treatments can be applied to tin dioxide in order to improve the selectivity (5). Tin dioxide has been used for gas detection in different forms: as bulk material from sintered powder, as thick films, and recently more and more as thin films, using microelectronic techniques.

Due to its electrical and optical properties (electrical conductor and transparent to visible light), $\mathrm{SnO}_{2}$ has been widely studied these last 20 years for optoelectronic applications (solar cells, LED, ...). For such applications, it has been prepared as thin films, particularly using the CVD technique. Only a few authors has been recently interested in chemical vapor deposition for gas sensors (6-7). Previous studies carried out in our laboratory (8) on $\mathrm{SnO}_{2}$ thin films elaborated by reactive evaporation and by CVD have shown the advantages of this last method. Indeed, the CVD technique allows us to modify the structural and the electrical properties of $\mathrm{SnO}_{2}$-thin films by the accurate control of the deposition parameters: temperature of deposition, pressure in the reactor, concentration and flows of the gases, deposition time, ...

\section{CVD installation}

The results from our first experiments with tin dioxide CVD have lead us to improvements in the experimental set-up. The main problems encountered were the control of the organometallic concentration and the repeatability due to gas flow in the reactor. We choose therefore to work at low pressure in order to overcome the problem of gas flow and gas diffusion in the proximity of the furnace, and to decrease the concentration of impurities in $\mathrm{SnO}_{2}$.

The $\mathrm{SnO}_{2}$ films with low impurities concentrations can be obtained through the oxidation of an organostannic compound, as it has been confirmed by Auger spectroscopy (8). The chosen organometallic precursor is dibutyltin diacetate (DBTD). It is an oily, air-stable, colorless liquid compound having a vapor pressure of $1 \mathrm{mbar}$ at $100^{\circ} \mathrm{C}$, which makes it convenient for transporting its vapor in a CVD process. A schematic representation of the CVD installation is shown in figure 1 .

Gas flows are regulated by four massflow meters from 0 to $1000 \mathrm{sccm}$. In lines 1 and $2, \mathrm{O}_{2}$ and $\mathrm{N}_{2}$ are conveyed to a gas mixer. In line 3 , the carrier gas $\mathrm{N}_{2}$ is bubbled in the DBTD solution, of which the temperature is regulated at $100^{\circ} \mathrm{C}$. Line 4 is used to purge the reactor before and after the process. The apparatus used for the generation of the DBTD in its vapor phase is heated up to $120^{\circ} \mathrm{C}$ in a drying-oven to avoid recondensation of the organometallic. The gases $\mathrm{N}_{2}, \mathrm{~N}_{2}+$ DBTD and $\mathrm{O}_{2}$ are mixed, and a small quantity of the reactive mixture is introduced into the CVD reactor by an inlet valve. The CVD reactor is a stainless steel cylinder with a volume of roughly 6 liters. A window on the upper side allows one to follow the $\mathrm{SnO}_{2}$ deposition during the process. The CVD furnace is placed in the middle of the reactor. It is a square brass block $8 \mathrm{~cm}$ large, which can be heated up to $600^{\circ} \mathrm{C}$ using electrical resistance heating.

The pressure in the cold wall reactor ranges from $10^{-1}$ to $10^{2} \mathrm{mbar}$. It is regulated by a butterfly valve. All the parameters of the reaction (gas flow, temperature of deposition, pressure) and the control of the electro-valves are fixed by a link to a central computer. This allows us to adopt a definite procedure and to improve the reproducibility of different runs carried out with the same parameters. A mass spectrometer is connected to the reactor and can be used to follow in situ the decomposition of the DBTD, and also to have a better understanding of the film growth mechanism. 


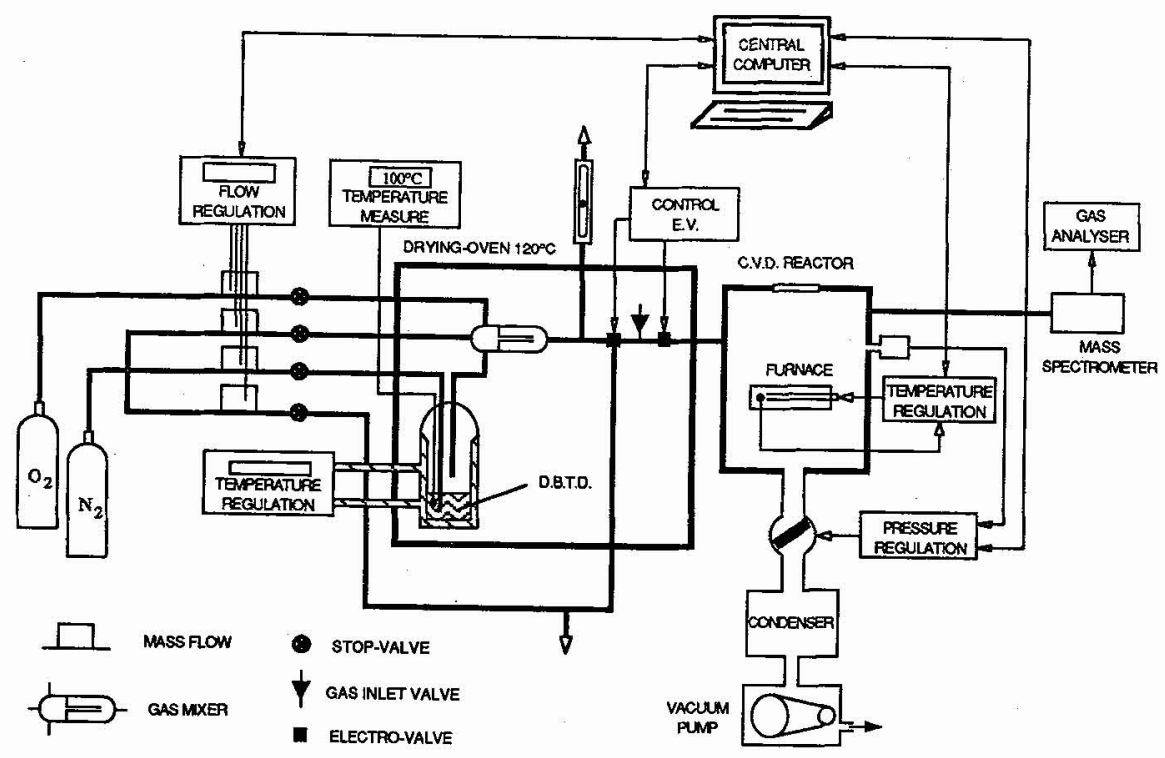

Fig. 1. - General view of the CVD installation.

\section{Conductivity testing bench}

Variations of conductivity of gas sensors are based upon adsorption and desorption mechanisms which occurs in a reversible manner at different temperatures, depending on the nature of the gas. This is the reason why particular attention has been given to the conductance measurements in the $200-500^{\circ} \mathrm{C}$ temperature range. A specific testing bench has been developed in our laboratory to measure the film conductance as a function of temperature under different gases (fig. 2).

Thin films are set in a measuring cell where the temperature and the gaseous atmosphere are controlled. Gases concentrations are obtained either from industrial gas $(\mathrm{CO}$, $\mathrm{CH}_{4}$ ), or by control of the partial pressure through permeation tubes of liquid coumpounds (alcohol, benzene). In this measuring cell, a brass block, heated by electrical resistances (up to $600^{\circ} \mathrm{C}$ ) is used to support thin films. The electrical measurement is carried out by means of two points with platinum wires. The resistance of the $\mathrm{SnO}_{2}$ film is calculated from the measurement of the electrical current through the film for a constant voltage.

For electrical measurements (fig. 3), the chosen substrate is alumina, which is a good electrical insulator. Two gold thin films electrodes are placed onto the substrate. The $\mathrm{SnO}_{2}$ film is then deposited above the electrodes. These conductance measurements have been previously confirmed by the four point probe technique (8).

The experimental procedure to obtain such curves is as follows: the $\mathrm{SnO}_{2}$ film is exposed in the measuring cell to a gas during 30 minutes at $500^{\circ} \mathrm{C}$. The conductance is recorded whilst the temperature decreases down to $100^{\circ} \mathrm{C}$ during a period of 30 minutes. The film is then exposed in the cell to pure air at $500^{\circ} \mathrm{C}$ during a further 30 minutes, in order to desorb the adsorbed species, and the conductance is again recorded during 30 minutes with decreasing temperature. Gas/air cycles are also executed. Electrical characteristics $G=f\left(T^{\circ}\right)$ are then recorded and plotted. This testing bench is controlled by a programmer which commands the opening and the closing of the electro-valve and temperature cycles. 


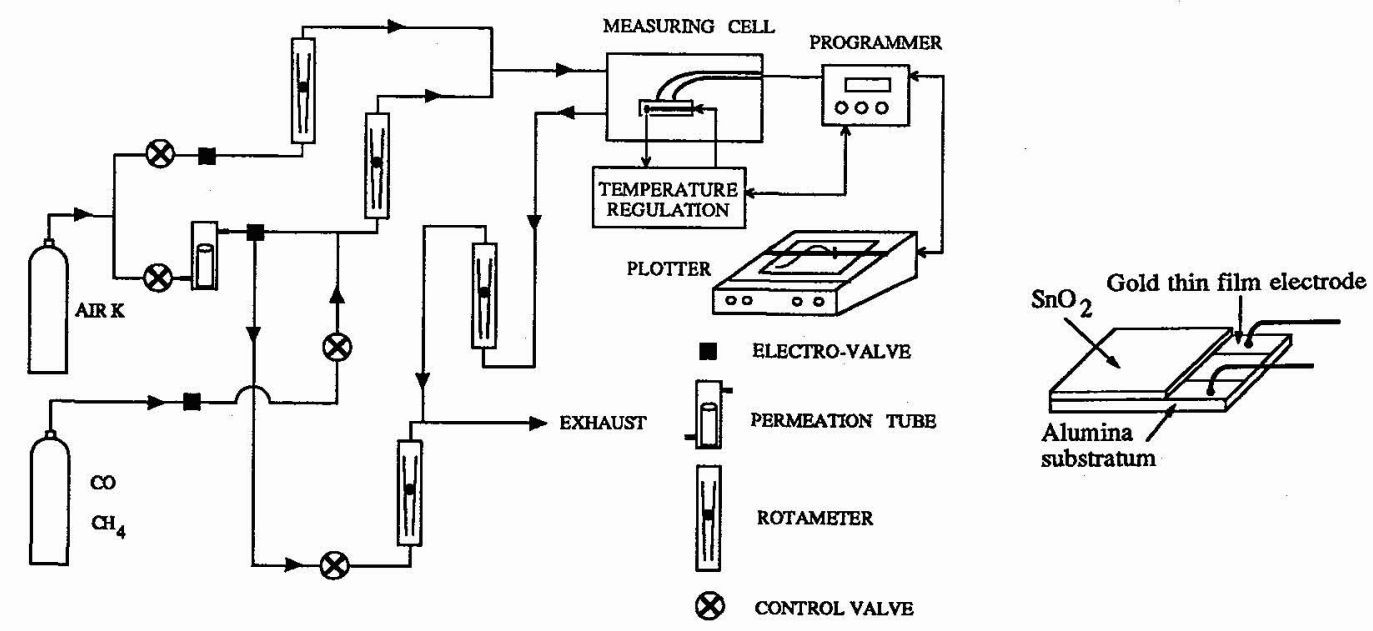

Fig. 2. - Conductivity testing bench.

Fig. 3. - Detail of the electrical contacts.

\section{Characterization}

A standard X-ray diffraction installation has been used to characterize $\mathrm{SnO}_{2}$ films. Measurements have been carried out on (100) silicium substrate, whose diffraction peak is located near $70^{\circ}$. It is necessary to have films with a sufficient thickness $(>1 \mu \mathrm{m})$ to obtain diffraction patterns with a good resolution. By an other method, thinner films (1000 $\AA$ ) have been studied with the low angle X-ray diffraction technique and the samples present the same diffractograms.

The $\mathrm{SnO}_{2}$ cassiterite structure has been confirmed (fig. 4). The relative intensities of the different peaks are the same as those of cassiterite. There is no preferential orientation of the crystal. The grain size is estimated from the broadening of the (110) peak in the X-rays diffraction spectrum.

Particular attention has been turned to the influence of annealing on the structural and electrical properties of the $\mathrm{SnO}_{2}$ films. One of the most evident effects of annealing on the structural properties of tin dioxide is the increase of the grain size. For example (fig. 5), before annealing, the average grain size of a $\mathrm{SnO} 2$ film is $50 \AA$. Successive annealings have been carried out on the same sample. After 1 hour at $600^{\circ} \mathrm{C}$, the mean size of the grains is $65 \AA$ and it growths up to $135 \AA$ after $1 \mathrm{~h} 30$ at $800^{\circ} \mathrm{C}$.

The structural aspect of the CVD films has been studied by scanning electron microscopy (fig. 6). SEM micrographs shows a compact structure, with a granular surface. The adherence to the silicium substrate seems to be good. It is also a way of measuring the thickness of the film. For instance, on this figure, it is possible to estimate the thickness as three microns. This value is in good agreement with those obtained by another way. Indeed, two other methods have been used for thickness measurements. Thickness can be evaluated by an optical method based upon the properties of tin dioxide (transparent to visible light), by an interferometric procedure. This method gives a good accuracy: $100 \AA$ for the thinner films $(<2000 \AA)$, and about $500 \AA$ for higher thickness. Another method, using the $\mathrm{SnO}_{2}$ film as a planar waveguide between two materials having lower optical indices, allows the determination of the refractive index and the thickness. These thickness measurements allows us to estimate the deposition rate: according to the deposition parameters, it ranges from 10 to $100 \AA$ Áminutes. 


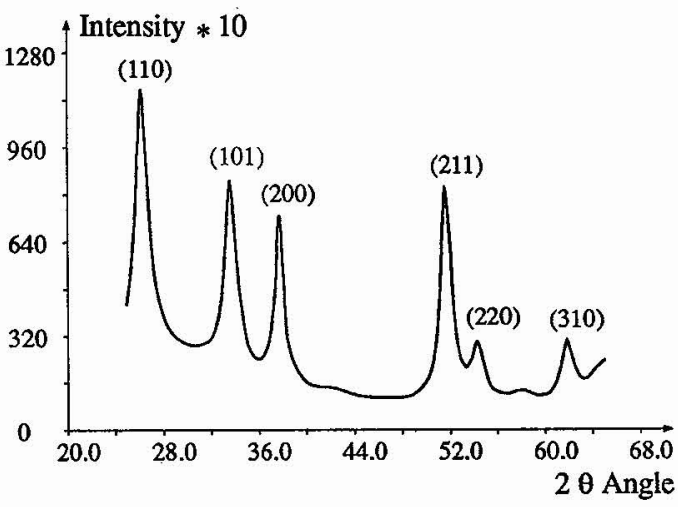

Fig. 4. - X-ray diffraction pattern of a CVD $\mathrm{SnO}_{2}$ film.

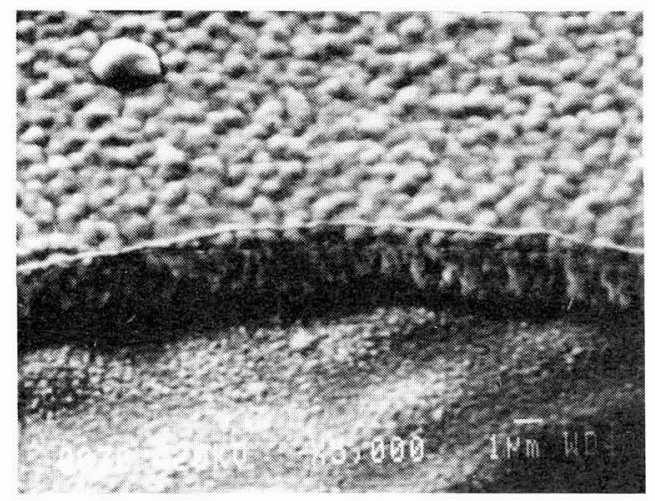

a.

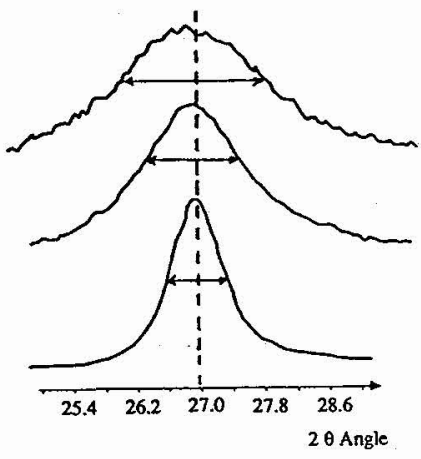

Fig. 5. - Influence of annealing on the mean grain size of CVD $\mathrm{SnO}_{2}$ film.

a. $\mathrm{L}=50 \AA$ without annealing

b. $\mathrm{L}=65 \AA$ annealed 1 hour at $600^{\circ} \mathrm{C}$

c. $\mathrm{L}=135 \AA$ annealed 1 hour at $600^{\circ} \mathrm{C}$, then 1 hour 30 at $800^{\circ} \mathrm{C}$

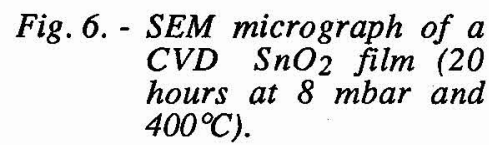

\section{Electrical results}

Electrical properties of CVD-SnO 2 thin films are particularly important for gas sensing applications. The conductivity testing bench described above can be employed to obtain $G=f\left(T^{\circ}\right)$ curves for different gases of various concentrations. The information given by these types of curves are: the value of the conductance in pure air at $500^{\circ} \mathrm{C}$, which is an intrinsic characteristic of the film; the sensitivity to a gas, which is calculated from the ratio $\left(\mathrm{G}_{\text {gas }}-\mathrm{G}_{\text {air }}\right) / \mathrm{G}_{\text {air }}$; the long term stability which can be observed during several weeks by continuous recording of $G=f\left(T^{\circ}\right)$ curves; the temperature of the maximum conductance which can be shifted depending upon the deposition parameters.

The value of the conductance measured at $500^{\circ} \mathrm{C}$ under pure air depends on the deposition parameters and can vary over several orders of magnitude. In fact, these variations seem principally to be connected to the thickness $d$ of the film. The influence of the thickness on the electrical conductance is reported in figure 7. An increase in the conductance versus thickness is always observed, whatever the deposition parameters may be. From these results, the values of the conductivity $\sigma$ of the different films can be calculated $(\sigma=\mathrm{G} / \mathrm{e})$ and it can also be observed that $\sigma$ increases with the thickness. This last result will be taken into account in the discussion. 
The influence of the deposition temperature has been studied in the range of 300 $600^{\circ} \mathrm{C}$. This is the temperature range in which the vapor phase reaction occurs between oxygen and the stannic compound (DBTD). The variation of the conductance with the deposition temperature is reported in figure 8, the other parameters (time, gas flows and pressure) being kept constant. A maximum of conductance can be observed for the deposition temperature of $480^{\circ} \mathrm{C}$. If we take into account the results of figure 7 , these variations of conductance seem to be connected to the thickness of the film, and especially to the deposition rate: this rate is highest at $480^{\circ} \mathrm{C}$.

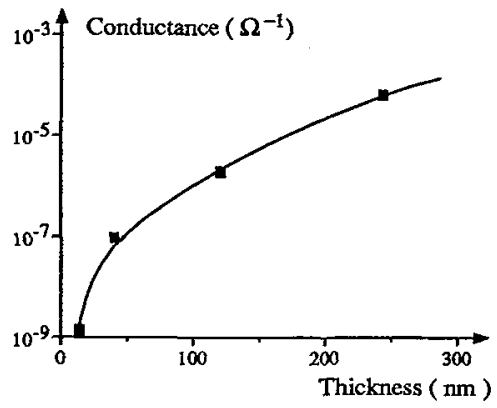

Fig. 7. - Influence of the thickness on the conductance.

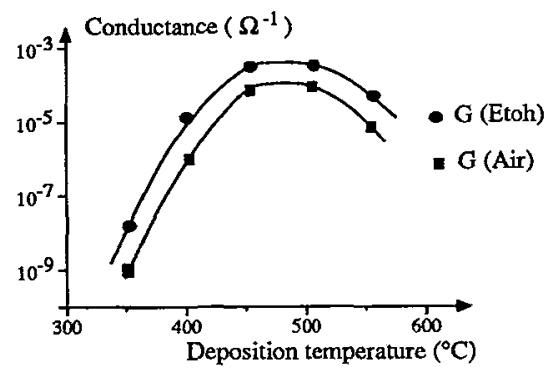

Fig. 8. - Influence of the deposition temperature on the conductance.

All the conductance measurements presented above are carried out on thin films which are considered as electrically stable. In effect, the stability of the electrical response of a gas sensor is an important parameter which must be controlled. The stability depends strongly on the annealing of the $\mathrm{SnO}_{2}$ films. An unannealed film shows a conductance which is always decreasing with time. After annealing, the conductance value is greatly diminished and there is no further variation. The gas sensor can be considered as stable. Two parameters are taken into account for annealing: duration and temperature. For a medium annealing temperature $\left(580^{\circ} \mathrm{C}\right)$, the conductance seems to tend to a limiting value versus annealing time (Fig. 9). On the other hand, (Fig. 10) for an annealing time of 1 hour, there is a continuous decrease of conductance with the annealing temperature up to $650^{\circ} \mathrm{C}$. The values reported in figures 9 and 10 have been measured at $500^{\circ} \mathrm{C}$ under pure air and for identical samples obtained at the deposition temperature of $400^{\circ} \mathrm{C}$.

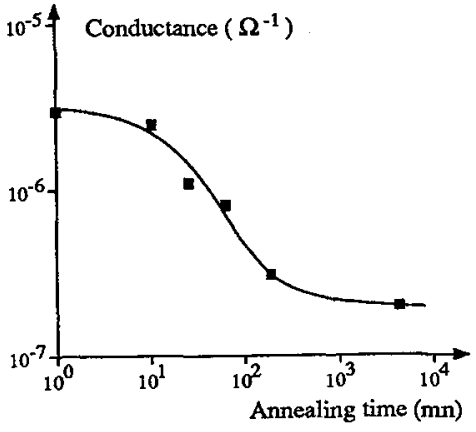

Fig. 9. - Influence of annealing time on the conductance.

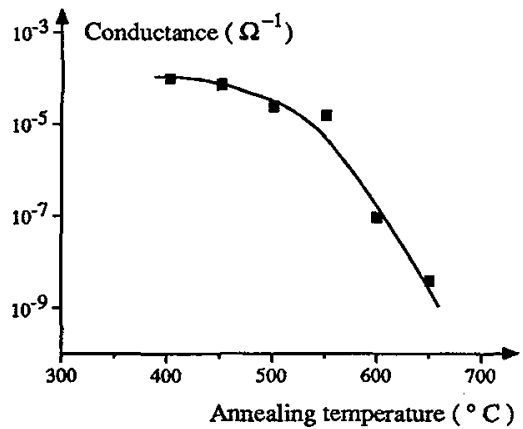

Fig. 10. - Influence of annealing temperature on the conductance. 
The characteristic $G=f\left(T^{\circ}\right)$ curves obtained for different gases are reported in figure 11. Altough the absolute curves are dependent upon the deposition parameters, their relative shapes are generally the same according to the nature of the gas: (a) a sharp peak near $300^{\circ} \mathrm{C}$ for alcohol, $\left(320^{\circ} \mathrm{C}\right.$ for a film deposited during 10 minutes at $400^{\circ} \mathrm{C}$ and at $1 \mathrm{mbar}$ ), (b) a smoother peak in the $350-450^{\circ} \mathrm{C}$ range for carbon monoxide, and on the contrary, a continuous increase of conductance for methane (c). For benzene (d), a slight maximum is present at around $500^{\circ} \mathrm{C}$ and it can only be observed with high temperature recordings. As it has been previously noticed, the value of conductance under pure air is closely dependent on the deposition parameters: for instance, $G=3.10^{-9} \Omega^{-1}$ for a film deposited during 30 minutes at $12 \mathrm{mbar}(\mathrm{d})$ and $5.10^{-6} \Omega^{-1}$ for a film deposited during 16 hours at $8 \mathrm{mbar}$ (c).

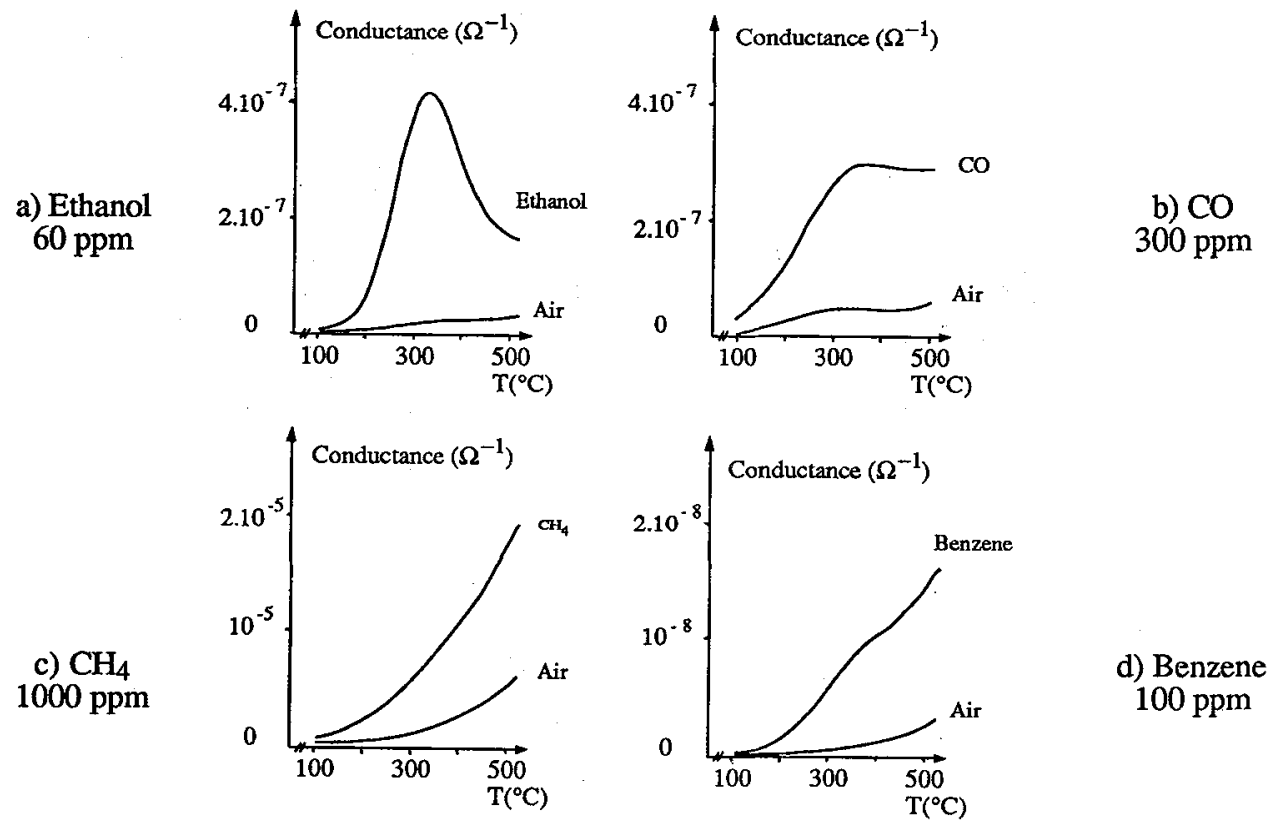

Fig. 11. - Characteristic $G=f\left(T^{\circ}\right)$ curves with different gases.

\section{Discussion and conclusion}

out.

Concerning the applications for gas sensors, different electrical results can be pointed

Firstly, the variations of conductance with the thickness of the film are difficult to explain, especially when the fact that the conductivity $\sigma$ varies is taken into account. This can be the result of our own measurements, with the two electrodes located above the $\mathrm{SnO}_{2}$ film. Moreover, it has been noticed that the best sensitivites are obtained with the thinner films (with deposition times of a few minutes), in comparison to the thicker films. This can be explained by the fact that there are two modes of conductions: a surface conductivity, depending on the adsorbed species, and a bulk conductivity, which is 'an intrinsic characteristic of the material. The thickness of the surface conductivity layer is given, in the Windischmann and Mark model (9), by a characteristic length, the depletion layer thickness $d_{s c}$. This characterises the depth into 
the solid which is depleted of conduction electrons as a result of the trapping of electrons in states localised at the surface.

Secondly, we can observe that, with the same sensitivities (i.e. $\left(G_{\text {gas }}-G_{a i r}\right) / G_{a i r}$, see figure 8), it is possible to change the value of the conductance over a very large range (from $10^{-9}$ to $10^{-4} \Omega^{-1}$ in pure air). This result is important insofar as industrial applications are concerned, since it is easier to measure high conductances. We can see how to take advantage of such a CVD method to prepare materials with special properties depending on the choice of the deposition parameters. This can also be applied for the improvement of the selectivity of gas sensors. Indeed, the deposition temperature has also an influence on the temperature of the maxium conductance observed with some gases: for instance, the conductance peak can be shifted by varying the deposition parameters. This constitutes an important way of investigation and we can hope to establish correlations between the deposition parameters and the evolution of the different shapes of the $G=f\left(T^{\circ}\right)$ curves observed under various gases.

Thirdly, it is interesting to note the influence of annealing on the electrical properties, i.e. the value of the conductance and its stability. These results must be correlated with the increase in the grain size. For a gas sensor, the stability of such polycrystalline materials is a major problem. Recently, Yamazoe (10) has shown that electrical stability seems to be linked to the grain size stability and this can be controlled by doping the starting powder used. In the present work, we have shown that the CVD technique allows us to prepare $\mathrm{SnO}_{2}$ films with various structural and electrical properties. This can be used to achieve reliable sensors, especially in regards of stability and also to have a better understanding of phenomena which occur in such materials, more particularly electrical conductivity mechanisms.

\section{References}

1- SEYIAMA T., KATO A., FUKIISHI K., NAGATINI M., Anal. Chem., 34, 1962, $1502-1503$

2- TAGUCHI N., Japanese patent, 1962, 4538200

3- MOSELEY P.T., TOFIELD B.C., "Solid State Gas Sensors", 1987, Adam Hilger, Bristol, England

4- PIJOLAT C., LALAUZE R., Sensors and actuators, 14 (1), 1988, 27-33

5- LALAUZE R., PIJOLAT C., Sensors and actuators, 5 (1), 1984, 55-63

6- BUTTA N., MELLI M., PIZZINI S., Sensors and actuators B, 2, 1990, 151-161

7- CAVICCHI R., SEMANCIK S. VETRONE J., CHUNG Y., Proc. Third Int. Meeting on Chem. Sensors, Cleveland, 1990, 337

8 BREUIL P., Thesis, Ecole des Mines de Saint-Etienne, 1989

9- WINDISCHMANN H., MARK P., J. Electrochem. Soc., 126 (4), 1979, 627-633

10- YAMAZOE N. Proc. Third Int. Meeting on Chem. Sensors, Cleveland, 1990, 24-26. 